Meta

Journal des traducteurs

Translators' Journal

\title{
Traduction et stéréotypie : quand le stéréotype détermine la facture du texte
}

\section{Saïd Mosbah}

Volume 45, numéro 3, septembre 2000

La traduction dans le monde arabe

URI : https://id.erudit.org/iderudit/003671ar

DOI : https://doi.org/10.7202/003671ar

Aller au sommaire du numéro

Éditeur(s)

Les Presses de l'Université de Montréal

ISSN

0026-0452 (imprimé)

1492-1421 (numérique)

Découvrir la revue

Citer cet article

Mosbah, S. (2000). Traduction et stéréotypie : quand le stéréotype détermine la facture du texte. Meta, 45(3), 458-464. https://doi.org/10.7202/003671ar
Résumé de l'article

Le texte du Petit Prince en arabe tunisien offre l'occasion de montrer comment l'usage des stéréotypes dans la langue d'arrivée peut servir de marqueurs de niveau de langue et d'éléments d'évaluation stylistique. C'est en partant d'exemples relevés dans le texte arabe dialectal, ne traduisant pas des stéréotypes figurant dans le texte français, que nous avons essayé de dégager les moyens mis en œuvre par le traducteur pour donner à son texte la facture voulue. 


\title{
Traduction et stéréotypie: quand le stéréotype détermine la facture du texte
}

\author{
SAÏD MOSBAH \\ Université de Tunis I, Tunis, Tunisie
}

\begin{abstract}
RÉSUMÉ
Le texte du Petit Prince en arabe tunisien offre l'occasion de montrer comment l'usage des stéréotypes dans la langue d'arrivée peut servir de marqueurs de niveau de langue et d'éléments d'évaluation stylistique. C'est en partant d'exemples relevés dans le texte arabe dialectal, ne traduisant pas des stéréotypes figurant dans le texte français, que nous avons essayé de dégager les moyens mis en œuvre par le traducteur pour donner à son texte la facture voulue.
\end{abstract}

\begin{abstract}
The text of "Le Petit Prince" ("The Little Prince") in Tunisian Arabic offers the opportunity to show how the use of stereotypy in the target language can serve as a language level marker and as a stylistic evaluation element. It is by examining examples from the Arabic text, which do not translate the stereotypes found in French, that we have tried to identify the means used by the translator to give his text the required construction.
\end{abstract}

\section{MOTS-CLÉS/KEYWORDS}

traduction, style, stéréotype, dialectal, tunisien

\section{Le Petit Prince en tunisien}

La situation linguistique en Tunisie se caractérise par une diglossie doublée d'un bilinguisme (arabe-français) qui touche en particulier les lettrés. Elle connaît une diglossie où figurent, d'une part, le littéral, langue officielle qui se propage surtout grâce à l'enseignement, et d'autre part, le dialectal, langue courante dans l'usage quotidien. Si le premier est un système fermé, dont la présence est plutôt écrite, le second est un système strictement oral, d'où sa grande ouverture (et sur le littéral et sur l'emprunt à d'autres langues en général) et sa variation régionale et sociale malgré une certaine tendance à la standardisation, laquelle n'aboutit pas, dans l'état actuel des choses, à son uniformisation.

Une telle situation fait de la traduction d'une ouvre étrangère dans le dialectal un acte original qui ne s'effectue pas sans difficultés, les rapports entre le littéral et le dialectal étant conflictuels, ce qui ne favorise pas une telle entreprise.

De plus, cette tentative de transposition impose au traducteur des difficultés de taille liées aux problèmes du passage d'un code oral à un code écrit. Parmi ces contraintes, figure, tant au niveau lexical que syntaxique, une grande variété régionale (archaïsmes, emprunts...) qui impose la recherche d'une forme standard accessible à tout lecteur. Sur le plan stylistique, l'absence de toute tradition de production littéraire écrite dans le dialectal et, par conséquent, l'absence de toute tradition de lecture, complique davantage la tâche du traducteur. 
Ainsi, s'aventurer dans ce sens, c'est œuvrer sur un terrain en friche où l'enjeu linguistique est très important: le choix stylistique doit faire émerger les potentialités expressives du dialectal, niveau de langue dont le traducteur valorise l'emploi.

Partant de la traduction en arabe tunisien, du Petit Prince d'Antoine de SaintExupéry par Hédi Balegh, nous essaierons de montrer comment le recours aux stéréotypes langagiers détermine le style du texte obtenu:

- en renforçant les marques spécifiques du dialectal (la fréquence des emprunts, la syntaxe propre, etc.),

- en faisant varier les formes stéréotypiques (expressions intensives, énoncés proverbiaux, etc.),

- en employant diverses expressions pour exprimer la même idée,

- en surchargeant le texte d'arrivée d'expressions non nécessaires à la traduction du texte de départ.

\section{Les emplois stéréotypiques dans le texte arabe}

\subsection{Le stéréotype comme marque spécifique au dialectal}

Dans cette situation de diglossie où la tradition de l'écriture en général est liée au littéral, les risques d'interférence linguistique entre les deux systèmes sont réels. Ainsi, le passage de l'oral à l'écrit devrait-il mettre en saillance les spécificités du dialectal tant syntaxiques que lexicales.

Les joncteurs sont l'une des marques de cette spécificité:

\section{Texte français}

(1) J'ai réussi à tracer mon premier dessin. (p. 4)
Texte arabe

/Ritmekkint be:Snsawwi:r ?عwwil taswi:ra

fi: je:ti:/

litt.: «J'ai pu dessiner le premier dessin de ma vie.» (p. 3)

L'équivalent dialectal verse le contenu sémantique de l'énoncé original dans une structure qui exprime la modalité du pouvoir. À la construction directe entre le semiauxiliaire et le verbe exprimant le procès, /nعz3imt ?insawwir/ 'pouvoir dessiner', le traducteur a préféré un autre élément verbal /Ritmekkint/ 'pouvoir', lequel, emprunté au littéral, impose dans sa structure d'origine l'emploi du joncteur /min $2 \varepsilon n /$. Son intégration fait appel, en dialectal, au joncteur /be: $/ /+$ verbe exprimant le procès.

(2) Je lui parlerais de bridge, de golf,

de politique et de cravates. (p. 6)
/... n $\varepsilon d \theta u: \div \varepsilon l g o l f ~ w \div \varepsilon l b r i: d 3$ w $\div \varepsilon s s i j \varepsilon: s s \varepsilon$ w $\div \varepsilon l$ krafata:t/ (p. 5)

La structure du verbe doublement transitif introduit le complément d'objet second au moyen de la préposition $/ \div \varepsilon-\div \varepsilon l /$. Il s'agit d'une forme tronquée de son correspondant littéral $/ \div \varepsilon$ n/. En réalité, le dialectal emploie trois formes équivalentes - $/ \div \varepsilon /, / \div \varepsilon n /$ et $/ \div 1 \varepsilon /$ - pour exprimer ce qui est rendu en littéral par deux joncteurs distincts $/ \div \varepsilon \mathrm{n} /$ 'de' et / $\div \varepsilon l \varepsilon: /$ 'sur'.

Au niveau lexical, l'ouverture sur l'emprunt est l'une des ressources enrichissantes que le traducteur déploie pour rendre compte de cette spécificité.

(3) J'étais irrité par mon boulon. (p. 32)
/Rilbulu:n nerfizni: barfa/ litt.: «Le boulon m’a énervé beaucoup.» (p. 31) 
Si le mot [bulu:n] n'a pas de correspondant en dialectal, le verbe /nerfiz/ peut par contre être traduit au moyen de plusieurs expressions dialectales. Toutefois, le choix se justifie ici par l'interaction linguistique entre les deux éléments empruntés, interaction qui donne plus de consistance à la facture dialectale.

(4) J'ai horreur des courants d'air. /me:ni mils 2ilku:rande:r/ (p. 40$)$ litt.: «Je ne supporte guère le courant d'air.» (p. 39)

Là aussi, là où le littéral aurait contourné le sens en se servant d'une expression équivalente, le dialectal recourt au terme le plus direct.

En outre, le lexique, comme lieu privilégié de fixation des stéréotypes, sert de moyen idéal pour ancrer le texte dans le dialectal:

(5) Quand je réussis enfin à parler, je lui dis... (p. 8) m'est revenu, je lui ai dit...» (p. 7)

/weqtilli: rza $\div$ li: $\int \varepsilon: h i d ~ l \varepsilon \div q$ al qutlu:.../ litt.: «Le temps où le témoin de la raison

L'énoncé d'origine encadre le procès du dire au moyen d'une conjonction temporelle "quand» corrélée à un adverbe temporel «enfin». Une telle structure rend compte de la portion de temps imprécise que prend l'étonnement ultérieur à l'accomplissement du procès. L'énoncé traduit reprend d'une manière différente cet encadrement temporel: le recours à l'expression idiomatique stéréotypique /wعqtilli: rza $\div$ li: $\int \varepsilon: h i d ~ l \varepsilon \div q a l /$ situe l'état d'étonnement entre deux repères séparés d'un vide au moyen d'un encadrement temporel où l'élément /rza $\div$ li:/ 'm'est revenu', renvoie rétrospectivement au moment d'interruption de la conscience:

quand enfin

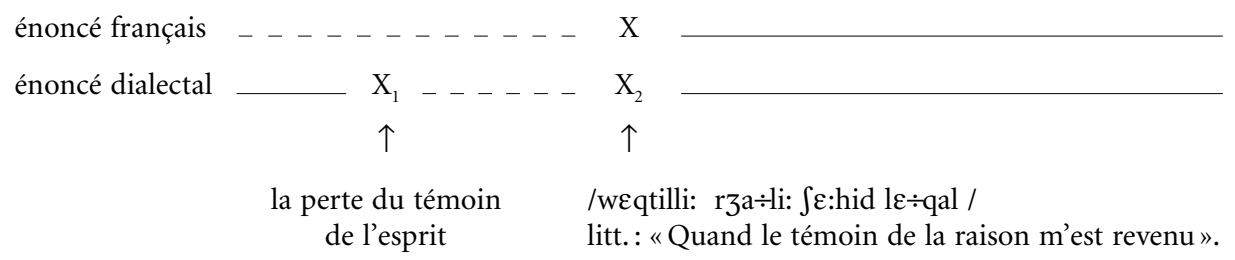

\subsection{La variation stéréotypique comme marqueur stylistique}

Cette valorisation des spécificités du dialectal prend parfois une dimension plus marquée et plus accentuée: le traducteur, au lieu de se contenter d'une seule forme lexicale, utilise la variation des formes stéréotypiques comme un véritable marqueur stylistique:

(6) Chez moi, c'est tout petit. (p. 12) comme une boîte». (p. 11)

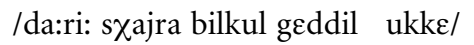
litt.: «Ma maison est toute petite tout

[Srajfa bilkul geddi nnet $\left.\int \varepsilon\right]$

litt. «Toute petite égale à une bouchée.»

(p. 11)

À la même expression d'intensité dans le texte de départ «c'est tout petit», correspondent deux expressions stéréotypiques où l'expression de l'intensité est rendue grâce à la redondance impliquée par le comparatif (cf. Mejri 1994) : 


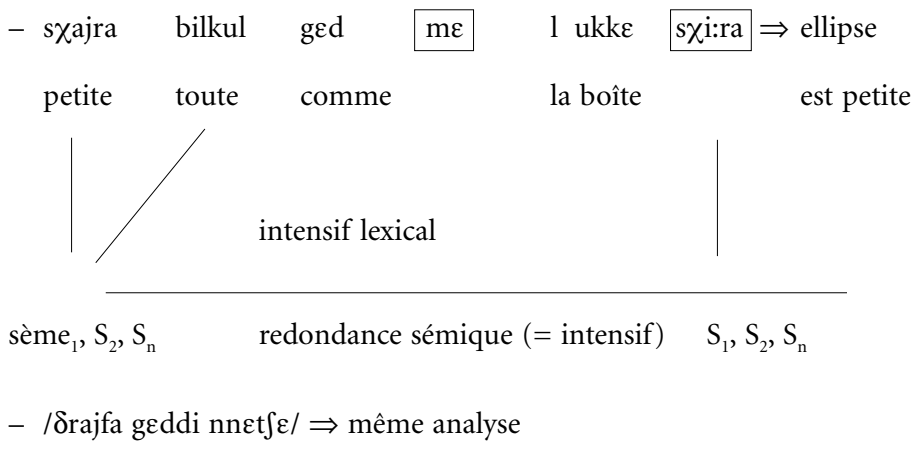

(7) Quand le mystère est impressionnant, on n'ose pas

/le Pile:he Pillella:h Pins wille zin désobéir. (p. 10) ou djin devant moi!» (p. 9)

gudde:mi/

litt.: «Il n' y a de dieu que Dieu! humain

Là où l'énoncé d'origine renvoie d'une manière assertive au référent situationnel, l'énoncé dialectal l'ancre dans le discours au moyen d'interjections spécifiques qui fonctionnent comme de véritables déictiques. L'embrayeur de personne [gudde:mi] 'devant moi' renforce la dimension discursive de l'énoncé.

(8) ...mais la fleur n'en finissait pas de se préparer à être belle. (p. 38)

et se fait-elle même.»
/Pinnawwa:ra ba:qi: ?it a $\delta \delta$ ir fi:ru: he wit gid fi: nefshe:

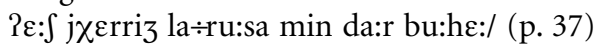
litt.: «La fleur encore prépare elle-même

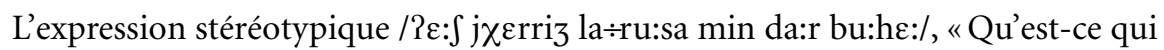
fait sortir la mariée de la maison de son père», surajoutée à l'énoncé initial, renvoie métaphoriquement au temps mis par la fleur pour se préparer. Son emploi ponctuel est restreint à la situation qu'elle commente. C'est le contexte qui la dote de sa fonction pragmatique: bien qu'elle reprenne connotativement le contenu sémantique de l'énoncé antérieur, elle ne peut le prendre totalement en charge.

(9)

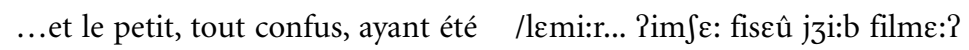

chercher un arrosoir d'eau fraîche, litt.: «Le prince est allé vite apporter de l'eau avait servi la fleur. (p. 40)

wlebbe: talab innawwa:ra:

et il a satisfait la demande de la fleur

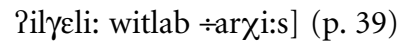

le cher a demandé le bon marché.»

L'énoncé proverbial renvoie au contexte antérieur (le service rendu par le petit prince). Il dénote la dépendance affective du petit prince à l'égard de la fleur; cette connotation est étroitement liée à la situation qu'elle évoque. Avec l'emploi de la dernière formule, aucune nouvelle information n'est ajoutée; ce qui change, c'est la marque stylistique. 


\subsection{L'expression du même contenu notionnel au moyen de plusieurs stéréotypes}

Nous avons déjà vu dans l'exemple (6) relatif à l'expression de l'intensité comment le même contenu notionnel est rendu au moyen de deux expressions stéréotypiques différentes. C'est un procédé mis au service du marquage stylistique comme le montrent (10) et (11):

(10) $[\ldots]$ c'était un monarque absolu. (p. 48-50)

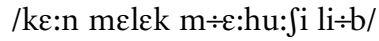 \\ «C'était un roi avec qui il n'y avait pas de \\ jeu.» (p. 47) \\ /Ri je: đibbe:ne \\ «Ouste, mouche!

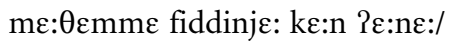 \\ Il n'y a dans le monde que moi.» (p. 49)
}

Dans la première expression, «absolu» est rendu grâce à «l'absence de jeu», ce qui est un signe de sérieux.

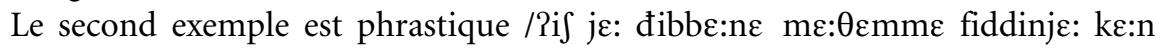

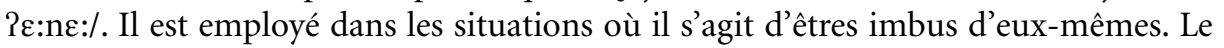
caractère dépréciatif est véhiculé par le terme «mouche».

La variation assure certes une élégance dans l'expression en permettant d'éviter les répétitions, mais l'auteur semble s'en servir surtout pour étaler la richesse lexicale du dialectal.

\subsection{La surcharge du texte dialectal d'expressions stéréotypiques}

Le recours à la densité des emplois stéréotypiques, là où les équivalents lexicaux suffisent pour rendre le sens global de l'énoncé d'origine, ne répond pas seulement à un choix ornemental de la part du traducteur, mais assure surtout des fonctions discursives évidentes.

\subsubsection{Les expressions surajoutées}

La surcharge stéréotypique permet, dans ce cas, de condenser les contenus diffus ou de les fixer dans des stéréotypes connus.

(12) Elle choisissait avec soin ses couleurs.

Elle s'habillait lentement.

Elle ajustait ses pétales. (p. 38) /texta:r fi: Relwe:nhe: wit rud be:lhe:

litt.: «Elle choisit ses couleurs et fait attention.»

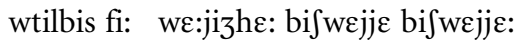

«Elle s'habillait de ses vêtements peu à peu.»

$\mathrm{t}$ ut fil atta

«Elle pose la pose»

kol warqa: fi: buq $\div$ ithe:/ (p. 37)

"chaque feuille à sa place.»

La comparaison des deux énoncés montre qu'à chaque énoncé dans le texte d'origine correspond un équivalent dans le texte d'arrivée. Seule l'expression /t ut fil atta/ n'a pas de correspondant, même si l'élément lexical /il atta/ reprend la totalité du passage et le condense dans une tournure intensive propre à l'arabe, qui consiste à employer un verbe suivi de son dérivé substantival. 
Ainsi, le contenu sémantique de l'unité stéréotypique surajoutée condense-t-il le sens des différentes opérations effectuées par le Petit Prince ainsi que celui de leurs circonstants.

(13) Ainsi, l'avait-elle bien vite tourmenté par sa vanité un peu ombrageuse. (p. 42)

/bde:t Rinnawwa:ra timriz fi:h litt.: «Commence la fleur à l'irriter titkabbir «se montre hautaine» wme $\div$ indhe:lihwe: wi:n jdu:r/ et n'a pas où l'air tourne.» (p. 41)

Il est évident que l'expression stéréotypique ajoutée, [me ûindhe:lihwe: wi:n jdu:r] ne traduit pas un contenu précis clairement formulé en L1 ; sa présence a plutôt une valeur stylistique puisqu'elle marque une façon de parler typiquement dialectale.

\subsubsection{Les expressions de renforcement}

Ces unités constituent une sorte de paraphrase stéréotypique intralinguistique.

Énoncé

(14) Mon ami ne donnait jamais d'explications. (p. 21-22)

(15) Il éclata brusquement en sanglots. (p. 35-36)

(16) Il raisonne un peu comme mon ami. (p. 65-66)

\section{Équivalents}

/meke:nja-tii:ni:hette:te:fsi:r/

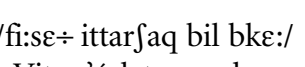

«Vite s'éclata en pleurs.» gorge »

/ifakkir ki:me: sa: ibne: «Il pense comme notre ami, ?issu:ke:rzi:/

l'ivrogne. »
Stéréotypes surajoutés

/le:sarf le: $\div \varepsilon \mathrm{dl} /$ «ni renvoi ni justice»

/bilyossa/ «une boule dans la

/Rimse:lul me:l «Il a perdu l'argent wiq $\div$ adlu: lihbe:1/ et il lui reste la folie.»

Toutes les expressions surajoutées reprennent les mêmes contenus sémantiques que ceux exprimés par les équivalents. Elles renforcent l'expressivité de leurs énoncés respectifs par leurs tonalités plus marquées. Si, dans les exemples (12) et (13), l'ajout d'expressions condense ce qui est diffus, dans les exemples (14) à (16), on peut se contenter de la séquence libre ou de la séquence figée. Le fait de les employer toutes les deux assure deux fonctions: une mise en relief due à la reprise et une recherche stylistique qui fournit au texte une teneur que les séquences libres sont incapables de garantir.

\subsubsection{Les expressions à équivalents unilexicaux non stéréotypiques}

Leur emploi permet d'éviter des confusions de sens dues aux confusions possibles entre sens compositionnel et sens non compositionnel:

(17) Tu as ri de toi-même. (p. 30)

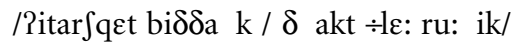
litt.: "Tu as éclaté de rire / tu as ri de toimême.» (p. 29)

L'énoncé / $\delta$ akt $\div$ le: ru: $i k /$, qui correspond à l'expression d'origine, est susceptible de deux interprétations:

- une lecture compositionnelle

- une lecture non-compositionnelle $\rightarrow$ «rire de toi-même»

$\rightarrow$ «se moquer soi-même» 


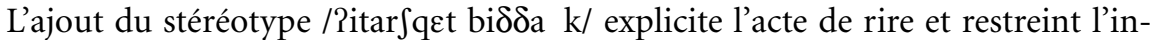
terprétation du deuxième segment à son niveau compositionnel.

(18) Le gros Monsieur rouge. (p. 36)

/wizhu: $\mathrm{mes}$ jitarfiq biddem/

litt.: «Son visage va éclater de sang.» (p. 35)

Là encore, l'équivalent unilexical, [?a mar] «rouge», aurait pu suffire :

razil ?a mar

homme rouge

Cependant, l'énoncé stéréotypique présente l'avantage d'une expression plus imagée.

\section{Conclusion}

L'usage de la stéréotypie, souvent perçu sous l'angle de la traductibilité, peut être étudié sous un angle stylistique.

Dans le cas du dialectal, l'unité unilexicale non stéréotypée peut être altérée par l'usage courant et perdre, par conséquent, une grande part de sa force expressive. Le recours à la stéréotypie lexicale permet, dans ce cas, de compenser ce manque et remotive, grâce aux fonctions multiples et variées auxquelles elle peut répondre, la potentialité expressive de la langue.

Le traducteur fournit ici la preuve que le recours à l'expression stéréotypique (idiomatique) est un outil linguistique idéal dont on peut se servir pour déterminer à la fois une appartenance linguistique et des choix stylistiques.

\section{RÉFÉRENCES}

Amossy, R. et P. A. Herschberg (1997) : Stéréotypes et clichés, Paris, Nathan, coll. «Nathan Université».

Baccouche, T. (1994): L'emprunt en arabe moderne, Tunis, Beit El Hikma - Carthage, IBLV Tunis I.

Clas, A., S. Mejri et T. Baccouche (1998): La mémoire des mots: Actes des Ves Journées du réseau LTT de l'AUPELF-UREF, Paris, AUPELF-UREF, coll. "Actualité scientifique».

Langue française (sept. 1999), 123, numéro spécial «La sémantique du stéréotype».

Mejri, S. (1994) : «Séquences figées et expression de l'intensité», Cahiers de lexicologie, 65-2.

- (1997): Le figement lexical: descriptions linguistiques et structuration sémantique, Tunis, $\mathrm{Pu}$ blications de la Faculté de Lettres de la Manouba.

Saint-Exupery, A. de (1997): Le petit prince, trad. en tunisien Hédi Balegh, Tunis.

SCHAPIRA, C. (1999): Les stéréotypes en français, Paris, Orphys. 